\title{
Preparation and performance evaluation of polyvinyl alcohol grafted polyacrylic acid for plugging
}

\author{
Jiangbo Liu ${ }^{1,2}$, Yi Ren ${ }^{3}$, Qin Zhang ${ }^{4}$, Xiangyun Wang ${ }^{5}$, Weichao Du ${ }^{5, *}$ \\ ${ }^{1}$ Oil \& Gas Technology Research Institute Changqing Oilfield Company, X i’an, Chian \\ ${ }^{2}$ National Engineering Laboratory of Tight Oil \& Gas Field Exploration and Development, Xi'an, Chian \\ ${ }^{3}$ Nanpu Operating Area, Jidong Oilfield, Tangshan, Hebei, China \\ ${ }^{4}$ Changqing Drilling Branch, CNPC Chuanqing Drilling Engineering Company Limited, Xi'an, Chian \\ ${ }^{5}$ Xi'an Key Laboratory of Tight oil (Shale oil) Development (Xi'an Shiyou University), Xi' an Shiyou University, Xi'an, 710065, China
}

\begin{abstract}
Well-leakage is a very difficult problem in the process of drilling at home and abroad. At present, it mainly relies on the cumulative effect of plugging slurry to implement plugging regardless of the number of times and costs. Commonly used plugging slurry and plugging technology are difficult to guarantee the success rate and scientific of plugging malignant leakage, which has an extremely bad impact on the development of the petroleum industry. In this study, With polyvinyl alcohol and acrylic acid as main agents, a plugging material of acrylic acid grafted polyvinyl alcohol was prepared, and the influence of the composition of the reaction system on the gel state of the system was studied. Through a series of singlefactor experiments, the best formula for preparing the plugging material was determined, polyvinyl alcohol and acrylic acid (specific gravity $1: 3)+$ crosslinking agent $(0.1 \mathrm{wt} \%)+$ initiator $(0.3 \mathrm{wt} \%)$. The gel plugging agent using above formula to prepare has good gel strength and suitable gel time, and has a good application prospect in solving the problem of lost circulation.
\end{abstract}

\section{Introduction}

During drilling operations, when the pressure of the drilling fluid column exceeds the formation pressure and the fractured formation is encountered, the drilling fluid can easily enter the pores and fractures of the formation, leading to the occurrence of lost circulation accidents [13]. As a more complicated accident in petroleum exploration and development, it needs to spend a lot of resources to deal with it. If it is not handled properly, it will lead to vicious leakage incidents and cause great environmental pollution problems. Adding plugging materials is a common treatment method in leakage accidents [4]. The plugging materials will have physical and chemical effects under the conditions provided by the formation, forming a network space structure, and forming a sealing layer at the formation cracks, thereby controlling leakage and ensuring stability Well wall [5]. The plugging materials include conventional plugging materials such as walnut shells, and chemical plugging materials that use the static force of the polymer at the interface and the intermolecular forces to bond the compounds to achieve the purpose of plugging [6]. In petroleum exploitation, Chemical gel is often used as a plugging material. When the plugging agent enters the formation, under the action of a certain temperature and pressure, various components interact [7]. Retention, accumulation, bridging, and cracking occur Filling and reinforcement, thereby improving the cementing and sealing pressure-bearing capacity of the gel in the leakage formation, achieving the purpose of sealing the leakage layer [8]. Among them, polymer gel plugging agent is an important branch of chemical gel plugging agent, compared with general plugging materials. The advantages are obvious, with: (1) low density is beneficial to the protection of oil and gas reservoirs; (2) gelling time is controllable, and the controllable time range is large, which is convenient for it to accurately reach the missing layer for plugging; (3) plugging The leaking slurry has strong fluid loss performance, and the formation of filter cakes can help stabilize the well wall; (4) It can be used in various applications and can block micro-fractures and relatively large fractures; (5) The gel plugging agent has good compatibility with the formation [9-11]. It will not affect the formation environment. And for large fractures in complex formations, traditional materials are prone to false plugging, and when polymer gel plugging agent is used to plug the leaky layer, polymer molecules and water When the molecules are in contact, the internal chemical bonds will be connected to each other [12]. A gel with a special three-dimensional network structure and high viscoelasticity is formed, and the number and types of intermolecular chemical bonds determine the shape and performance of the polymer spatial structure.

\footnotetext{
* Corresponding author: duweichao@xsyu.edu.cn
} 


\section{Experimental}

\subsection{Materials}

Polyvinylamide was purchased from Shandong Yuhuang New Energy Technology Co., Ltd. Polyvinyl alcohol was purchased from Tianjin Zhiyuan Chemical Reagent Co., Ltd. Acrylic acid was purchased from Luoyang Haohua Chemical Reagent Co., Ltd. N. N-methylene bisacrylamide was purchased from Tai'an Changxing Engineering Materials Co., Ltd.

\subsection{Gel Preparation}

Preparation of $10 \%$ polyvinyl alcohol solution: $15 \mathrm{~g}$ polyvinyl alcohol and $150 \mathrm{ml}$ distilled water were added into a three neck flask, which was put into a water bath pot and stirred at $95{ }^{\circ} \mathrm{C}$ until polyvinyl alcohol was completely dissolved. A certain amount of acrylic acid was added into the beaker, and $40 \%$ sodium hydroxide solution was slowly added in the cold-water bath to neutralize the acrylic acid, so that the neutralization degree of acrylic acid reaches the preset value. An appropriate amount of polyvinyl alcohol solution was added to the neutralized acrylic acid solution, and then the crosslinker N, N-methylene bisacrylamide, initiator ammonium persulfate and sodium sulfite were added to the mixed solution in turn. After stirring the mixed solution evenly, it was put into a water bath for heating, and the gelation of the reaction was observed.

\section{Results and Discussion}

\subsection{Effect of Polyvinyl alcohol concentration on gel-forming condition}

The composition of the gelling system is as follows: the concentration of polyvinyl alcohol was $7 \%, 8 \%, 10 \%$, $11 \%, 12 \%$ and $13 \%$ respectively, the mass ratio of polyvinyl alcohol to acrylic acid was $1: 3$, the neutralization degree of acrylic acid was $60 \%$, the dosage of initiator (ammonium persulfate and sodium sulfite) was $0.3 \%$ of the mass sum of polyvinyl alcohol and acrylic acid, and the dosage of the crosslinker (N,N-methylene bisacrylamide) was $0.1 \%$ of the mass sum of polyvinyl alcohol and acrylic acid, and the reaction temperature was $100{ }^{\circ} \mathrm{C}$. The gel-forming conditions of different systems were shown in Table 1.

Table 1 The gel-forming conditions of system with different Polyvinyl alcohol concentration

\begin{tabular}{ccc}
\hline $\begin{array}{c}\text { Polyvinyl alcohol } \\
\text { concentration }\end{array}$ & $\begin{array}{c}\text { Gelation time } \\
(\mathrm{h})\end{array}$ & $\begin{array}{c}\text { Gel } \\
\text { strength }\end{array}$ \\
\hline $7 \%$ & 7 & $\mathrm{I}$ \\
$8 \%$ & 7 & $\mathrm{I}$ \\
$10 \%$ & 6.5 & $\mathrm{I}$ \\
$11 \%$ & 4.5 & $\mathrm{I}$ \\
$12 \%$ & 5.5 & $\mathrm{I}$ \\
$13 \%$ & 5.5 & $\mathrm{I}$ \\
\hline
\end{tabular}

From Table 1, the gelation time of each system was $7 \mathrm{~h}, 7$ $\mathrm{h}, 6.5 \mathrm{~h}, 4.5 \mathrm{~h}, 5.5 \mathrm{~h}$ and $5.5 \mathrm{~h}$ respectively. When the concentration of polyvinyl alcohol in the reaction system was no more than $10 \%$, the gelation time of the reaction system was relatively short. However, when the concentration of polyvinyl alcohol increases in the reaction system, the gelation time of the reaction system gradually decreased. The reason was that when the dosage of polyvinyl alcohol in the reaction system was small, the contact probability between polyvinyl alcohol and the active radical produced by the initiator was small, which made the reaction slower and the gelation time of the reaction system longer. When the dosage of polyvinyl alcohol was large, the chance of collision between polyvinyl alcohol molecules and active radicals was significantly improved, which speeded up the reaction rate of the reaction system. When the concentration of polyvinyl alcohol was higher than $12 \%$, the gelation time of the gel system was no longer affected by the amount of polyvinyl alcohol. The gel strength of each reaction system reached grade I, and $11 \%$ polyvinyl alcohol concentration was selected as the best concentration for subsequent experiments.

\subsection{Effect of initiator on gel-forming condition}

The composition of the gelling system was as follows: the concentration of polyvinyl alcohol was $11 \%$, , the mass ratio of polyvinyl alcohol to acrylic acid was $1: 3$, the neutralization degree of acrylic acid was $60 \%$, and the dosage of the crosslinker (N,N-methylene bisacrylamide) was $0.1 \%$ of the mass sum of polyvinyl alcohol and acrylic acid, the initiator systems were ammonium persulfate (oxidation), sodium sulfite (reduction), ammonium persulfate-sodium sulfite (oxidationreduction), the reaction temperature was $100^{\circ} \mathrm{C}$. The gelforming conditions of different systems are shown in Table 2 .

Table 2 The gel-forming conditions of system with different initiator systems

\begin{tabular}{ccc}
\hline initiator system & $\begin{array}{c}\text { Gelation } \\
\text { time }(\mathrm{h})\end{array}$ & Gel strength \\
\hline ammonium persulfate & 4 & $\mathrm{I}$ \\
sodium sulfite & Not gelled & $\mathrm{D}$ \\
ammonium & & \\
persulfate-sodium & 2 & $\mathrm{I}$ \\
sulfite & & \\
\hline
\end{tabular}

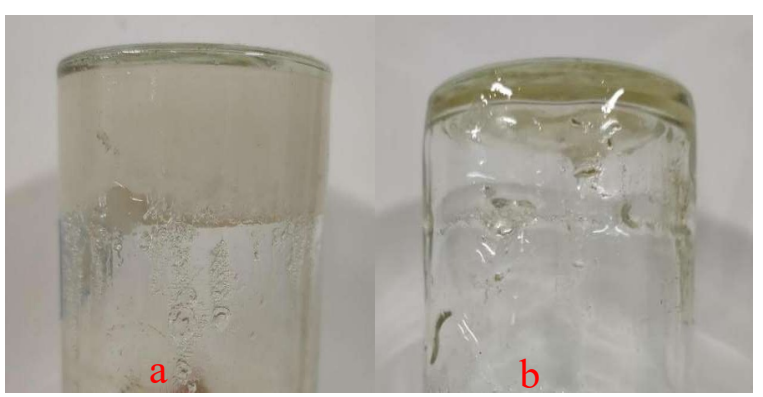




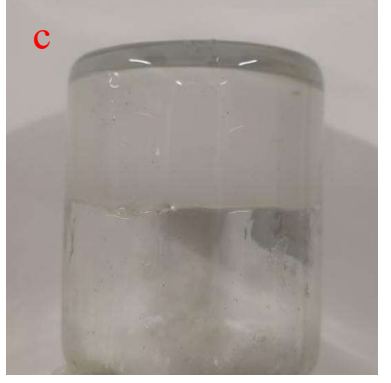

Figure 1 The gel-forming morphology of systems with initiator systems: (a)ammonium persulfate, (b)sodium sulfite, (c)ammonium persulfate-sodium sulfite

Ammonium persulfate $\left(\left(\mathrm{NH}_{4}\right)_{2} \mathrm{SO}_{4}\right)$ is an inorganic oxidizing initiator, which is used for aqueous solution polymerization and emulsion polymerization. When the temperature is higher than $60^{\circ} \mathrm{C}$, ammonium persulfate may decompose effectively, and the decomposition product is free radical, which can effectively initiate the polymerization reaction. Sodium sulfite $\left(\mathrm{Na}_{2} \mathrm{SO}_{3}\right)$ is usually used as a reducing initiator. According to the experimental data in Table 2, when ammonium persulfate was used as initiator, the gelation time of the reaction system was longer, which was 4 hours, and the strength reached grade I. When the initiator was sodium sulfite, the reaction system was basically not gelled. When ammonium persulfate sodium sulfite was used as initiator, the gelation time of the reaction system was 2 hours, the gelling time was appropriate and the strength was excellent. Therefore, ammonium persulfate- sodium sulfite was used as initiator for subsequent experiments.

\subsection{Effect of initiator concentration on gel- forming condition}

The composition of the gelling system was as follows: the concentration of polyvinyl alcohol was $11 \%$, the mass ratio of polyvinyl alcohol to acrylic acid was $1: 3$, the neutralization degree of acrylic acid was $60 \%$, the dosage of the crosslinker (N,N-methylene bisacrylamide) was $0.1 \%$ of the mass sum of polyvinyl alcohol and acrylic acid, and the dosage of the initiator (ammonium persulfate-sodium sulfite) was $0.1 \%, 0.3 \%, 0.4 \%, 0.6 \%$ of the mass sum of polyvinyl alcohol and acrylic acid, the reaction temperature was $100{ }^{\circ} \mathrm{C}$. The gel-forming conditions of different systems are shown in Table 3.

Table 3 The gel-forming conditions of system with different initiator concentration

\begin{tabular}{ccc}
\hline $\begin{array}{c}\text { initiator concentration } \\
(\%)\end{array}$ & $\begin{array}{c}\text { Gelation } \\
\text { time }(\mathrm{h})\end{array}$ & Gel strength \\
\hline 0.1 & 1.5 & $\mathrm{I}$ \\
0.3 & 1.0 & $\mathrm{I}$ \\
0.4 & 1.0 & $\mathrm{I}$ \\
0.6 & 1.0 & $\mathrm{I}$ \\
\hline
\end{tabular}
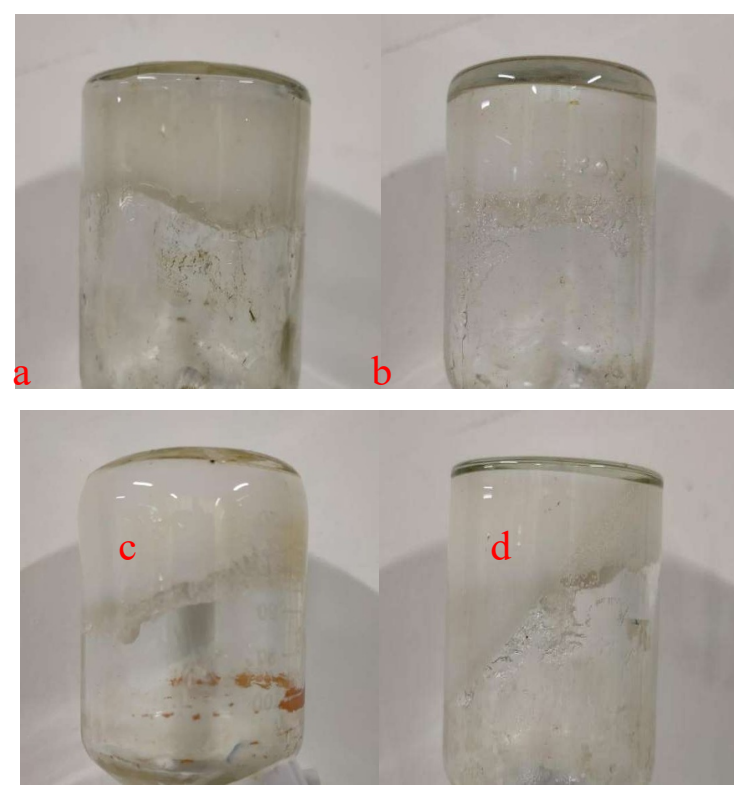

Figure 2 The gel-forming morphology of systems with different initiator concentration: (a) $0.1 \%$, (b) $0.3 \%$, (c) $0.4 \%$, (d) $0.6 \%$.

It can be seen from Table 3 that when the amount of initiator was $0.6 \%$ of the mass sum of the two monomers, the crosslinking reaction rate was too fast and the gelation time was too short. When the amount of initiator was $0.1 \%, 0.3 \%$ and $0.4 \%$ of the mass sum of polyvinyl alcohol and acrylic acid, the gelation time of the reaction system was 1 hour. The reason was that when the amount of initiator was relatively small, there were relatively few active free radicals produced by the initiator in the reaction system, so that the polymerization rate was slow. As shown in Figure 2.2, compared with the reaction system with other concentrations of initiator, the system with initiator concentration of $0.3 \%$ of the sum of the mass of two monomers had the greatest gel strength. The reason was that the concentration of living radicals in the reaction system affected the crosslinking density and affect the gel strength.

\subsection{Effect of neutralization degree of acrylic acid on gel-forming condition}

The composition of the gelling system was as follows: the concentration of polyvinyl alcohol is $11 \%$, the mass ratio of polyvinyl alcohol to acrylic acid is $1: 3$, the neutralization degree of acrylic acid is $0,60 \%, 92.5 \%$, $97.5 \%$, the dosage of the crosslinker (N,N-methylene bisacrylamide) is $0.1 \%$ of the mass sum of polyvinyl alcohol and acrylic acid, and the dosage of the initiator (ammonium persulfate-sodium sulfite) is $0.3 \%$ of the mass sum of polyvinyl alcohol and acrylic acid, the reaction temperature is $100{ }^{\circ} \mathrm{C}$. The gel-forming conditions of different systems were shown in Table 4 . According to the table, when the neutralization degree of acrylic acid was $0 \%$, the gelling time of the reaction system was too fast. When the neutralization degree was $60 \%$, when the neutralization degree of acrylic acid is $92.5 \%$ and $97.5 \%$, the gelation time of the reaction system 
was long, and the gel strength reached I grade. The main reason is that when the neutralization degree of acrylic acid was relatively small, the concentration of acrylic acid in the reaction system is high, and the internal reaction of acrylic acid was easy to self-react, resulting in the acceleration of chemical reaction and the final gelation time of the gel system. When the neutralization degree of acrylic acid was large, the concentration of -COONa generated by neutralization reaction between acrylic acid and sodium hydroxide was too high, which had an effect on the rate of chemical reaction and made the system of gelation time too long. Therefore, $60 \%$ is the best neutralization degree of crosslinking reaction.

Table 4 The gel-forming conditions of system with different neutralization degree of acrylic acid

\begin{tabular}{ccc}
\hline $\begin{array}{c}\text { Neutralization degree } \\
(\%)\end{array}$ & $\begin{array}{c}\text { Gelation } \\
\text { time }(\mathrm{h})\end{array}$ & Gel strength \\
\hline 0 & 0.5 & $\mathrm{I}$ \\
60.0 & 1.0 & $\mathrm{I}$ \\
92.5 & 5.0 & $\mathrm{I}$ \\
97.5 & Not gelled & $\mathrm{C}$ \\
\hline
\end{tabular}
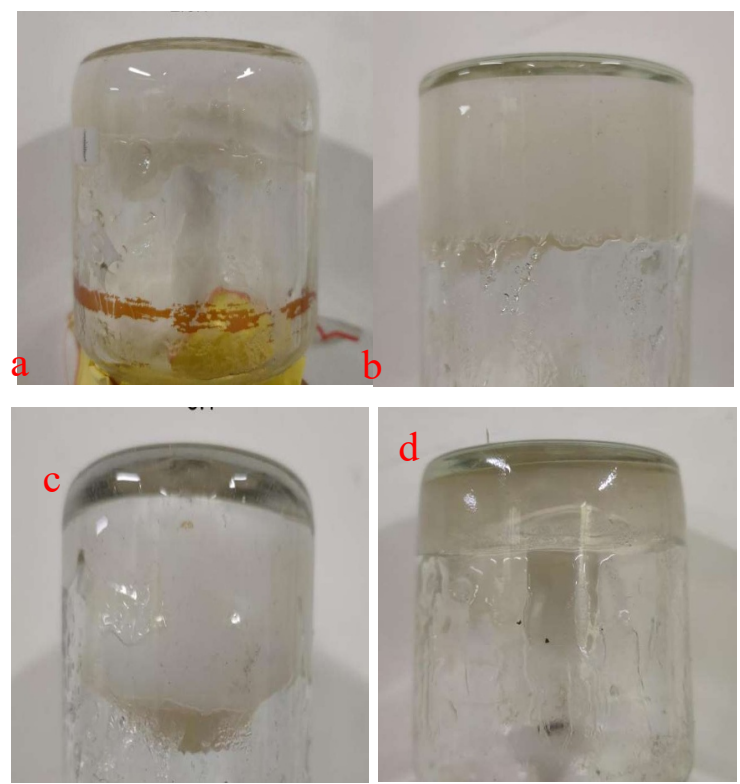

Figure 3 The gel-forming morphology of systems with different neutralization degree of acrylic acid: (a) 0 , (b) $60 \%$, (c) $92.5 \%$, (d) $97.5 \%$.

\section{Conclusion}

In this paper, a resin plugging agent was synthesized by solution polymerization with acrylic acid and polyvinyl alcohol as polymerization monomers, ammonium persulfate and sodium sulfite as redox initiator and N, Nmethylene bisacrylamide as crosslinking agent. At the same time, the experimental conditions were optimized by controlling variable method. The synthesis process was as follows: the specific gravity of acrylic acid and polyvinyl alcohol was $4: 1$, the dosage of initiator was $0.3 \%$ of the mass sum of acrylic acid and polyvinyl alcohol, the dosage of crosslinking agent was $0.1 \%$ of the mass sum of acrylic acid and polyvinyl alcohol, the neutralization degree of AA was $60 \%$, and the water bath was set at $70{ }^{\circ} \mathrm{C}$. The plugging agent prepared by the above experimental conditions has excellent gel strength and suitable gelation time, and has a good application prospect in solving the well leakage problem.

\section{Acknowledgements}

The work was supported financially by Shaanxi Provincial Key Research and Development Program (2019GY-136) and Youth Innovation Team of Shaanxi University. And we thank the work of Modern Analysis and Testing Center of Xi'an Shiyou University.

\section{References}

1. Y. S. Wang, H. Zhang, J. F. Fu. New progress in chemical plugging technology $[\mathrm{J}]$. Science and Technology Vision, 2015, 1(1): 9-10.

2. J. W. Fang, Z. K. Lu, Z. He. Application of chemical gel plugging agent pressure-bearing plugging technology in Shunbei 3 well [J]. Drilling Fluid and Completion Fluid, 2017, 34 (6): 13-17.

3. X. C. Cao, B. R. Li, Y. Qin. Research and application of polymer gel plugging agent $[\mathrm{J}]$. Contemporary Chemical Industry, 2015, 44(11): 65-67 .

4. X. P. Wang, Y. X. Zheng, C. X. Zhang. Preparation of P(AA-AMPS) water-absorbent resin and determination of salt resistance in high temperature environment [J]. New Chemical Materials, 2019, 47(3): 185-188.

5. X. R. Li, Y. Zhang, N. Yang. Performance evaluation of pre-crosslinked gel microsphere plugging agent $[\mathrm{J}]$. Oilfield Chemistry, 2016, 33(4): 581-584.

6. J. Y. Mou, J. J. Shao, B. Y. Lu. Plugging performance of acid fracturing temporary plugging agent for fractured-vuggy reservoirs $[\mathrm{J}]$. China Science and Technology Papers, 2016, 11(3): 258-262

7. Y. Y. Liu, Y. H. Zhang, H. Zhang. Experimental study and field application of high-strength fiber composite temporary plugging agent [J]. Petrochemical Technology, 2017, 24(8): 101-104.

8. Wilson A. Thermally stable fluid-loss pill performs better than guar-base gels [J]. Journal of Petroleum Technology, 2013, 65(11): 134-136.

9. P. Gamage, J. P. Deville, J. Shemian. solids-free fluid-loss pill for high-temperature reservoirs [J]. SPE Drilling \& Completion, 2014, 29(1): 125-130.

10. C. L. Hu, P. X. He, G. C. Jiang. Self-degradable leak prevention and plugging system for workover operations [J]. Acta Petrolei Sinica, 2016, 37(2): 150157.

11. R. J. Liu, D. Lu, D. Wang. Research progress of insitu intercalation polymerization to prepare 
nanocomposites [J]. Shandong Chemical Gong, 2016, 45(15): 52-54.

12. H. Jia, H. Chen, B. Chen. Leak prevention mechanism and application of elastic liquid rubber plug for well repair [J]. Acta Petrolei Sinica, 2018, 39(3): 349-356. 